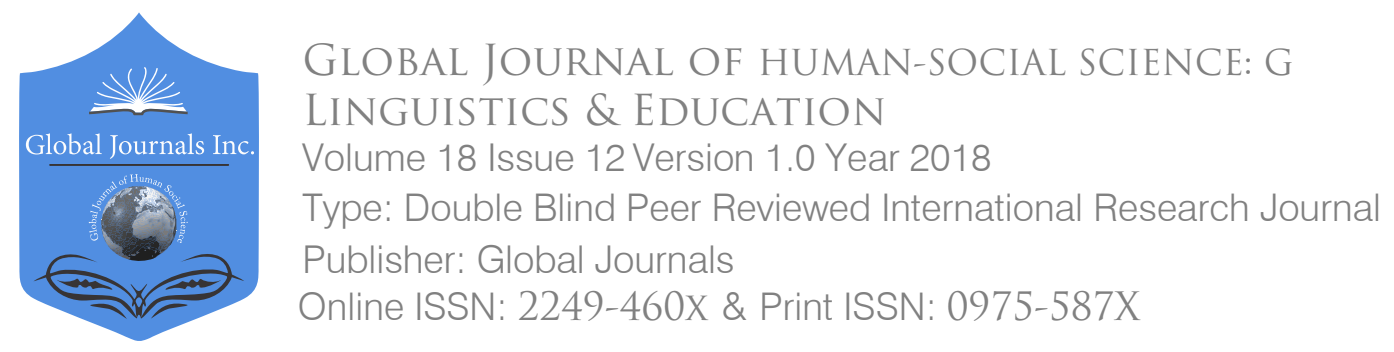

\title{
On Persian Japanese Intonation
}

By Norouzi Tayebeh

University of Tsukuba

Abstract- The aim of this study is to investigate learners' acquisition of Japanese lexical accent in different prosodic environments. Nineteen Iranian learners and one Japanese native speaker participated in the experiment and were asked to pronounce a dialogue using the same three-mora non-word in three different positions in the dialogue. The results show that native speaker pronounce the non-word with three possible accent types: Atamadaka-gata (i.e., the first mora has a high pitch $(H)$ and all subsequent morae have low pitches (L)), nakadaka-gata (i.e., one or more than one mora that is neither the first nor the last within that word has a high pitch) and heiban-gata (i.e., the first mora has a low pitch and all subsequent morae have a higher pitch, and the pitch gradually lowers). However, accent realization does not change in different prosodic environments. On the other hand, when analyzing the Iranian learners' pronunciation, a total of seven accent types were recognized, and the results demonstrate that accent realization differs by prosodic environment: while LHL (an accent form like nakadaka-gata) appears frequently in a focal environment, LHH (an accent form like heiban-gata), which is similar to a Persian accent, is realized more in other environments such as neutral or post-focal environments. The above result suggests that since, at the beginning of conversation sentences and in focal environments, the learners' degree of consciousness is higher than for the whole conversation, L1 interference is less likely to occur.

Keywords: japanese accent, japanese intonation, japanese prosody, iranian learners.

GJHSS-G Classification: FOR Code: 339999

Strictly as per the compliance and regulations of:

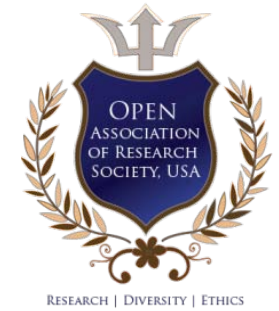

(C) 2018. Norouzi Tayebeh. This is a research/review paper, distributed under the terms of the Creative Commons AttributionNoncommercial 3.0 Unported License http://creative-commons.org/licenses/by-nc/3.0/), permitting all non-commercial use, distribution, and reproduction in any medium, provided the original work is properly cited. 


\title{
On Persian Japanese Intonation
}

\author{
Norouzi Tayebeh
}

Abstract- The aim of this study is to investigate learners' acquisition of Japanese lexical accent in different prosodic environments. Nineteen Iranian learners and one Japanese native speaker participated in the experiment and were asked to pronounce a dialogue using the same three-mora non-word in three different positions in the dialogue. The results show that native speaker pronounce the non-word with three possible accent types: Atamadaka-gata (i.e., the first mora has a high pitch $(\mathrm{H})$ and all subsequent morae have low pitches (L)), nakadaka-gata (i.e., one or more than one mora that is neither the first nor the last within that word has a high pitch) and heiban-gata (i.e., the first mora has a low pitch and all subsequent morae have a higher pitch, and the pitch gradually lowers). However, accent realization does not change in different prosodic environments. On the other hand, when analyzing the Iranian learners' pronunciation, a total of seven accent types were recognized, and the results demonstrate that accent realization differs by prosodic environment: while LHL (an accent form like nakadaka-gata) appears frequently in a focal environment, LHH (an accent form like heiban-gata), which is similar to a Persian accent, is realized more in other environments such as neutral or post-focal environments. The above result suggests that since, at the beginning of conversation sentences and in focal environments, the learners' degree of consciousness is higher than for the whole conversation, L1 interference is less likely to occur.

Keywords: japanese accent, japanese intonation, japanese prosody, iranian learners.

\section{INTRODUCTION}

S ince Japanese uses pitch to mark certain morae in utterances, it is considered a pitch accent language. It is very similar to Persian, as Persian uses pitch accents in its into national system. However, there are fundamental differences between the two languages. In this section, we provide a brief overview of Japanese prosody, highlighting the similarities and differences between Japanese and Persian.

First, Japanese, like Persian, only has two levels for prosodic organization: accentual phrase (AP) and intonation phrase (IP). AP is a sequence of low $(L)$ and high $(H)$ tones and has only one type of pitch accent. One or more APs make up the larger unit, IP, which is followed by a boundary tone (i.e., a rise or fall in pitch that occurs in speech at the end of each IP).

According to Japanese lexical accent characteristics, the pitch rises at the AP head, and if there is an accento-kaku, the pitch falls immediately after. This accento-kaku is discriminable and has been identified as the most important feature of the Japanese accent. Therefore, the existence or position of this accento-kaku creates more than one accent pattern.

Author: University of Tsukuba. e-mail: tyb_nrz@yahoo.com
Accent patterns in Japanese may be described as either kifuku-shiki (accented) or heiban-shiki (unaccented). Kifuku-shiki is further divided into atamadaka-gata (i.e., the first mora has a high pitch $(\mathrm{H})$ and all subsequent morae have a low pitch (L)), nakadaka-gata (i.e., one or more than one mora that is neither the first nor the last within that word has a high pitch), and odaka-gata (i.e., the first mora has a low pitch and all subsequent morae have a higher pitch, and the pitch falls suddenly when followed by certain particles). However, there are no subcategories to heiban-shiki. When contrasting heibanshiki with the above subcategories of kifuku-shiki, it is typical to use the word heiban-gata (i.e., the first mora has a low pitch and all subsequent morae have a higher pitch; the pitch falls gradually).

In a stress accent language like Persian, the accent of nouns, adjectives, and most adverbs falls on the final syllable of the prosodic word (hereafter, "word") and thus, the accented syllable position is predictable in most words. In addition, Persian has the concept of nuclear pitch accent (hereafter "NPA"). In other words, only the final AP of an utterance or IP is perceived as more prominent by the listener. Only the final AP has a low boundary tone while other APs have a high boundary tone (Sadat-Tehrani 2007). NPA, which is also known as a sentence accent, is absent in Japanese.

A third difference between the two languages that is related to the above concept is the case of focus realization. Japanese, like Persian, applies pitch range expansion in order to mark focus. Focal prominence has a remarkable effect on the following component as well as on the focused component itself. In other words, focal prominence brings prosodic subordination to the following APs. In Japanese, this subordination may delete the tone sequence that marks the boundary between two APs, but it does not delete the tone sequence of accent, and, actually, the post-focal accents are maintained. In Persian, on the other hand, the NPA falls on the focused component, and a complete dephasing occurs in the following APs. Since Persian uses NPA and Japanese does not, Persian learners of Japanese may find it difficult to realize accents in positions where an NPA is not present.

The above differences may cause language interaction during language acquisition; that is, Persian speakers' first language (L1) may interfere with their pronunciation of Japanese as a second language. Since pronunciation is greatly affected by L1 and since no explicit and systematic prosody education is provided to learners of Japanese, it is expected that learners will not 
be able to pronounce Japanese sentences while consciously maintaining the accent of words in various prosody environments.

Given the above information about the Japanese lexical accent (hereafter "accent"), it has been assumed that the topic of accent has long been a complex issue in Japanese language education; many studies have been conducted to investigate the realization and perception of the Japanese accent by learners of Japanese. The results have demonstrated that although nakadaka-gata is the most difficult accent type to perceive, most words are realized in the pattern of nakadaka-gata, in particular the pitch fall after the second to last mora of a word. However, only a few studies have focused on the effect of prosodic environment on the realization of accent.

To fill this gap, this study aims to investigate how different prosodic environments influence the realization of accent in Iranian learners of Japanese. We hope to clarify not only the details of Iranian learners' accent realization, but also its acquisition in various prosodic environments.

\section{Purpose and Hypotheses}

This study aims to investigate how Persian learners of Japanese realize accent in different prosodic environments. Given the above information, in this research, we will test the following hypotheses:

1. The tendency of accent realization will differ between APs receiving NPA (when the prosodic environment is strong) and APs not receiving NPA (when prosody is neutral or weak).

2. The accent rules of Persian will be introduced with respect to word accent, and accents will thus be placed at the end of the word.

\section{EXPERIMENT}

\section{a) Material}

Based on the characteristics mentioned in section 1, previous studies investigating Japanese learners' accent realization have focused on the accuracy of accent type by examining readers' reading of word lists (Sukegawa 1999, Nakato 2001). However, words are not usually uttered in isolation and since prosody elements such as sentence structure and intonation influence accent, the realization of accent is considered to differ in the case of reading a sentence than a single word. However, it is considered difficult to use free utterances as material for analysis and comparison, as the utterance amount, vocabulary, prosodic environment, etc., differ for each utterer (Ayusawa 2003).

Ayusawa (2003) thus proposed a test to investigate accent acquisition status with respect to nouns. She suggested setting up a dialogue in which the target nouns would be situated at several locations throughout the dialogue. If a noun is pronounced correctly in all locations, it can be inferred that the accent type of this noun has been acquired. Moreover, even when the accent type is not correct, if the same pitch pattern is used in different sentences, it can be surmised that the utterer is conscious of word accents.

In this study, based on Ayusawa (2003), a short dialogue was designed to test whether accent realization differs between different prosodic environments. We constructed a dialogue in which three positions were used as the target prosodic environments: environment 1 is a prominent location; environment 2 is a neutral location; and environment 3 is a post-focal location. Twenty non-words were then used to determine the lexical accent realization by Iranian learners. We applied non-words to control the effect of word frequency or possible acquisition on the realization of accent. The target dialogue is provided below:

\section{A: Osake desu ka?}

\section{Is that sake?}

$B$ : Osake janakute, (non-word) desu.

It's not osake, it's (non-word).

A: Sou desuka. (non-word) wa, dare no (non-word) desuka?

I see. Whose (non-word) is that (non-word)?

(The underlined positions are focused.)

\section{b) Subjects}

Nineteen Iranian learners of Japanese whose native language is Persian participated in the experiment. The subjects ranged in age from 20 to 23 and had a basic proficiency level. A female native speaker of Tokyo Japanese also participated as a control target.

\section{c) Procedure}

First, before the main experiment, participants signed a letter of consent in which they were fully informed about the purpose of the experiment and were assured that their voices would not be used for nonacademic purposes. Then, to avoid unnatural pronunciation, the subjects were asked to memorize the dialogue until they could say it by heart and become accustomed to the experiment environment. They were then asked to repeat the dialogue using the same nonwords in the target locations each time. Finally, they were paid for participating in the experiment. Subjects' utterances were recorded in a sound studio and the data were digitized.

\section{d) Measurements}

For the phonological description, two trained native speakers were paid to evaluate the non-word accent realized by Iranian learners. To reduce the influence from factors other than the target environment as much as possible and also to reduce the burden on the judges, the dialogues pronounced by the learners 
were edited so that only sentences including the nonwords remained while the other parts were excluded.

The native speakers were asked to label the accent of target non-word sets in each dialogue using $\mathrm{L}$ or $\mathrm{H}$. For convenience, only the pitch tracks of the target location were extracted and the time between these positions was removed. The native speaker judgments were conducted individually, and a kappa coefficient test found that the coincidence rate between judges was $k=.76$. Thus, it was considered that there was sufficient reliability. Discrepancies between evaluators were settled through consultation.

For the acoustic analysis, the pitch tracks were extracted using Praat software ver. 6.0.43 (Boersma \& Weenink 2010).

\section{Results}

Table 1 shows the accent realization tendency of the native speakers by environment. Three accent types, "HLL," "LHH" (flat plate type), and "LHL" were confirmed. "HLL" (80\%) was considerably more common than "LHL" (10\%) and "LHH" (10\%). However, each non-word was realized with the same accent type in all environments.

Table 1: Accent types and their frequency observed in the utterances of native speakers

\begin{tabular}{|l|l|l|l|l|}
\hline \multicolumn{5}{|c|}{ Accent type (\%) } \\
\hline Environment & HLL & LHL & LHH & Total \\
\hline 1 focus & $16(80)$ & $2(10)$ & $2(10)$ & $20(100)$ \\
\hline 2 neutral & $16(80)$ & $2(10)$ & $2(10)$ & $20(100)$ \\
\hline 3 post-focal & $16(80)$ & $2(10)$ & $2(10)$ & $20(100)$ \\
\hline total & $48(80)$ & $6(10)$ & $6(10)$ & $60(100)$ \\
\hline
\end{tabular}

Table 2: Accent types and their frequency observed in the utterances of learners

\begin{tabular}{|c|c|c|c|c|c|c|c|c|}
\hline \multicolumn{7}{|c|}{ Accent type (\%) } \\
\hline Environment & LHL & HLL & LLH & HHH & HLH & LLL & LHH & Total \\
\hline focal & $148(38.95) \mathbf{\Delta}$ & $80(21.05) \mathbf{\Delta}$ & $73(19.21) \mathbf{\Delta}$ & $34(8.94) \mathbf{\Delta}$ & $19(5) \mathbf{\Delta}$ & $0 \nabla$ & $26(6.86) \nabla$ & $380(100)$ \\
\hline neutral & $48(12.63) \nabla$ & $32(8.42) \nabla$ & $19(5) \nabla$ & $3(0.79) \nabla$ & $2(0.52) \nabla$ & $2(0.52)$ & $274(72.10) \mathbf{\Delta}$ & $380(100)$ \\
\hline post-focal & $28(7.36) \nabla$ & $38(10) \nabla$ & $0 \nabla$ & $0 \nabla$ & $1(0.26) \nabla$ & $7(1.84) \mathbf{\Delta}$ & $306(80.52) \mathbf{\Delta}$ & $380(100)$ \\
\hline total & $224(19.64)$ & $150(13.15)$ & $92(8.07)$ & $37(3.24)$ & $22(1.92)$ & $9(0.79)$ & $606(53.15)$ & $380(100)$ \\
\hline
\end{tabular}

\section{Discussion}

In section 4 , the accent tendencies of native speakers and learners were described based on the auditory impression of native speakers. In this section, we compare the utterances of the Japanese native speaker with the utterances of Persian learners and describe how the prosodic environment affects the realization of accent. Before analyzing the prosody realization in each utterance, it is necessary to explicate the prosody of Japanese related to the sentences applied in this study. In section 5.1, we first summarize the prosodic features of the potential accent type of the word and the prosodic features of the dialogue in standard Japanese. We then describe the accent
Table 2 shows Iranian learners' accent realization tendency by environment. As can be seen, overall, seven accent types ("LHH" (3.14\%), "HLH" (1.92\%), "LHL" (19.64\%), "HLL" (13.15\%), "LLH" (8.07\%), "HHH" (3.24\%) and "LLL" (0.79\%)) were confirmed. Among these, the realization frequency of "LHH" (53.15\%) was the highest.

A chi square test was conducted to examine whether there was any significant difference between the frequency of the above seven accent types depending on the environment, and a significant difference was observed $\chi^{2}(12)=558.207, p<.01$ Cramer's $V=$ 0.495). Therefore, residual analysis was conducted (Table 2). The results demonstrate that in environment 1 , "LHL" (38.95\%), "HLL" (21.05\%), "LLH" (8.94\%), and "HHH" (5\%) occurred more than other accent types, while the occurrence frequency of "LHH" (6.86\%) which was generally overwhelmingly high, was significantly low. In environment 2, "LHH" (72.10\%) occurred more frequently, indicating that the frequency of other accent types was significantly reduced. In environment 2 , as in environment 3, "LHH" (80.52\%) occurred the most while the frequency of other accent types decreased.

realization of the native speaker and learners observed in each prosodic environment.

a) Japanese Prosody

As defined by Matsuzaki and Kawano (2010: 34), "The arrangement of relative height and strength which is arbitrarily decided as a social custom in relation to each word, is called 'accent." However, the rules of arrangement of this height and strength differ depending on languages and dialects. There are two rules for the accent type of the Tokyo dialect: (1) the heights of the first and second morae differ; (2) once the tone falls, it does not rise again. In Tokyo dialect, $n+1$ accent types are potentially allowed for $\mathrm{n}$-morae words. However, there is not an equal number of words in each accent type. For example, in three-mora words there are 
theoretically four accent types of accent: atamadakagata (indicated like "HLL"), nakadaka-gata (indicated like "LHL"), and odaka-gata and heiban-gata (indicated like "LHH"). However, in actuality, their frequencies are not equivalent (Matsuzaki \& Kawano 2010).

On the other hand, it is said that the pronunciation of loanwords that have not yet been fully assimilated into the language reflect the accent system of the language in a simplified form, and such a word is pronounced with a specific accent type (Kubono \& Ota 1998). Specifically, in the Tokyo dialect, there is a rule that, for loanwords, "Accento-Kaku is applied to the third mora counted from the leftmost edge of a prosodic word" (Kubono \& Ota 1998: 81). Although loanwords were not used in this research, if we consider the target words as new loanwords for which the pronunciation of the original word is unknown, it is expected that they would be pronounced as HLL (atamadaka-gata) types by native speakers of the Tokyo dialect.

As mentioned above, environment 1 was the environment with focus. The focal point does not normally change the accent type, but it is one of the elements of focus in which the pitch range of the focused phrase is expanded (Kori 1997). Therefore, it can be assumed that "HLL" is environmentally friendly for environment 1, that environment 2 is a neutral environment but "HLL" can easily be applied as in environment 1, and that environment 3 is an interrogative question sentence, and comprises the environment immediately after the interrogative word in which prominence is easily placed.

Another feature of the focus is that the AP after the focal point is reduced and its independence disappears. In other words, the focused AP integrates with the immediately following $A P$ to form one IP. Accordingly, it is assumed that in dareno 'whose' (interrogative word), prominence is placed on the interrogative, the pitch range of the interrogative is expanded, the accent realization of the following nonwords is reduced, and one IP is formed. It is thought that in this way, the accent is weakened. Therefore, it can be assumed that the accent of the word in environment 3 is realized as "HLL" as in environments 1 and 2. In other words, non-words will be realized in the same accent type in all three environments.

b) Accent realization by the native speaker and learners

First, we will describe the tendency of prosody realization in each environment based on the native speaker's typical pitch patterns (Figures 1 to 3). Then, we will describe the tendency of prosody realization in each environment based on the learners' typical pitch patterns (Figures 4 to 12) For convenience, we extracted environments 1 to 3 and deleted pauses from the utterances. The horizontal axis represents time (seconds), while the vertical axis represents height; that is, the fundamental frequency (FO) of the sound. The scale of the height is indicated by a semitone value (st) based on $100 \mathrm{~Hz}$. Similar to other languages, the pitch differs between men and women (Ayusawa 1991).

\section{i. Native speaker's prosody}

Since environment 1 is the environment in which prominence is placed, as can be seen from Figures 1 to 3 , the pitch range of non-words is expanded. The pitch suddenly descends immediately after the mora on which the accento-kaku is placed. Similarly, in environment 2, the pitch suddenly falls immediately after the mora on which the accento-kaku is placed and does not rise to the end of the AP, including the particle "wa." In environment 3, prominence is placed on the question word dareno 'whose', and the AP, including the nonword, is weakened. Finally, "ka" of the final mora is realized with a rising tone. As mentioned earlier, in this experiment, the native speaker pronounced non-words as "HLL," "LHL," and "LHH." Of these, the "HLL" type was the most common. However, in all environments, words were realized with similar accent types.

\section{ii. Learners' prosody}

The non-words used in this study may be pronounced with various accent types by individuals. Specifically, as mentioned earlier, for three-syllable words, there are theoretically four possible accent types: "HLL," "LHL," "LHH" (odaka-gata), and "LHH" (heibangata). However, if the same non-words are realized with similar accents in various environments and realized at an appropriate height according to the environment, it can be said that, overall, accent and intonation have been acquired.

In accent realization by learners, "LHH" and "LLH" are similar to the Persian accent, but "LHH" (53.15\%) occurred many times, while the incidence of "LLH" (8.07\%) was extremely low.

In environment 1, non-words are pronounced with emphasis at the beginning of an independent phrase, despite appearing after the phase "osake janakute." As mentioned in the section 4, environment 1 has many variations of accent type as individual differences are large. Examining the learners' pitch patterns (Figures 4 to 12), we may observe that the accent tendency differs among individuals. There are also accent patterns such as "HLH," "LLH," and "HHH" that violate the accent rule of the Tokyo dialect among the seven accent types that have been confirmed. Overall, "LHL" (38.95\%) and "HLL" (21.05\%) are the most frequent. However, regardless of accent type, the pitch rage is sufficiently expanded. In other words, since in Persian, as in Japanese, prominence is realized by expansion of the pitch range, positive transfer allows learners to pronounce accented words with a focus at an appropriate degree of realization.

In the neutral utterance of environment 2, the accent types ("LHL," "LLH," "HLL," "HHH," "HLH") that occurred frequently in environment 1 decreased 
significantly, whereas in environment 1, the less frequent "LHH" was significantly higher than the other accent types $(72.10 \%)$.

Environment 2 appears at the beginning of the sentence and forms an independent AP followed by the particle "wa." The accent of the target word of environment 2 should be realized using the same type of accent as in environment 1. However, as can be seen from Figures 4 to 12, two patterns were used. These are a pattern (Figures 4 to 7 ) in which the same accent type as environment 1 was realized and a pattern in which an accent type different from environment 1 was realized (Figures 8 to 12).

Regarding the first pattern, since the learners were already conscious of the word accent, they used the same accent type as for environment 1. However, for the second pattern, no appropriate rise is seen in the phrase head, and the pitch is realized in a nearly flat form just before the end of the phrase. As described in the results, this pattern was judged to be $\mathrm{LHH}$.

On the other hand, a certain pattern can be seen in the tone at the end of the phrase. As observed in Figures 4 to 12, at the end of the phrase, Iranian learners (1) prominently pronounced the particle "wa" at the end of a phrase (Figures 4, 5, 7, 8, 9, 11), (2) give a rise to particle "wa" at the end of the phrase (Figure 6), or (3) lengthen particle "wa" at the end of the phrase (Figure 11). This can be regarded as a "habit of learners who rebuild new phrases by resetting every word" (Matsuzaki 2001: 239). However, the accent of the nonword in environment 2 is influenced by the phrase boundary tone and becomes "LHH."

Based on the above, when we summarize the procurement situation of environments 1 and 2, if words are pronounced with a similar accent type in both environments, and if the end of the sentence falls in tone, then it can be said that, on the pitch curve, the prosody became similar to that of the native speaker. Examining the learners' pitch patterns, prosody such as that above can be said to be realized only in S1 and S2 (Figures 4 and 5), and Japanese prosody can be learned.

In the next step, we see the intonation of interrogative sentences and the realization of accent weakening.

As confirmed in Table 2, after the focus of environment 3, "LHH" and "LLL" occur most frequently.

Environment 3 is an interrogative. In Japanese interrogatives, (1) the focus is placed on the interrogative, (2) the words in AP immediately following the interrogative word are subordinated until the end of the sentence, and finally, (3) the final mora of the IP rises.

As shown in Figures 4 to 12, focus was placed on interrogatives in the utterances of all members except learner 12, and non-words immediately following an interrogative word weakened until the end of the sentence. The accents of non-words pronounced in environment 3 were judged as "LHH."

In order to examine whether this is due to the influence of Persian prosody, we will first discuss the intonation of Persian sentences. As mentioned in SadatTehrani (2007), for Persian interrogatives, like in Japanese, prominence is placed on the interrogative word, the pitch range of the interrogative word is expanded, and the following AP is weakened. In addition, the sentence final tone of the simple interrogative is high, while the sentence final tone of the interrogative with a question word is low.

If Persian prosody is at work, it would be expected that learners' 'whose (non-word) is that?' would be realized by a falling tone. However, as seen in Figures 4 to 12, most of the Iranian learners were able to realize this question using a correct tone: that is, the rising tone. Specifically, the pitch range of dare 'who' was expanded due to prominence, and the AP of the non-word immediately after it was weakened. Finally, the end of the sentence was realized with a rising tone.

On the other hand, the final tone of the interrogative question sentence may be a cause of the "LHH" in environment 3. As mentioned above, the final tone of Japanese interrogatives is a rising tone, and the rise usually occurs at the last mora of the sentence. However, the rise of interrogatives by Iranian learners began before the final mora. Thus, in environment 3 , the non-words were realized with $\mathrm{LHH}$. In pronouncing dare 'who', there were cases in which the pronunciation was followed by a pause between the interrogative word dare 'who' and the target non-words, and cases in which the pronunciation was continued without pauses, as well. However, in both cases, the accent of nonwords was realized in a weakened form.

From the above, it can be said that beginner learners are able to acquire the final tone of Japanese interrogatives. However, since the accent of environment 3 is realized in a type different from environments 1 or 2 and in a form that rises toward the end of the sentence, it can be assumed that the accent type of the word is also influenced by the prosodic environment in environment 3. Therefore, the learners have already acquired intonation and are conscious of intonation, but they are not conscious of accent.

Ayusawa (1993) pointed out that the intonation of interrogatives is acquired in the following seven stages:

1. Step of pronouncing like L1 intonation.

2. Acquiring rising tone and pronouncing all interrogatives in the rising tone

3. Acquiring intonation of interrogatives with question words

4. Acquiring the final rising tone of interrogatives desuka? and masuka? 
5. Level of acquiring discrimination between nakadakagata and heibangata

6. Acquiring rising tone at the end of words with accent type -2 (i.e., the accento-kaku falls on the second-to-last mora).

7. Acquiring the rising tone when the word ends with $n$ or a long vowel.

Given the above acquisition stages, it can be assumed that the rising tone at the end of the sentence is acquired earlier than accent. Our study confirms this assumption. Iranian learners have acquired the correct final tone of interrogatives as well as dephasing the words immediately after the focus. However, they have not yet acquired the correct accent.

Utsugi (2004) noted that the Japanese accent is ignored, regardless of the presence or absence of the focus in learners' utterances, and the peak of the pitch appears on the second mora of the phrase as a whole. Similar results have been found for Brazilians in Sukegawa (1999) and for Koreans in Nakato (2001). From this, it can be considered that "LHL" is relatively frequently common in Japanese utterances by foreigners, and one interpretation of this is that the "LHL" has been formed based on learners' intermediate language strategy. However, this study shows that "LHL" occurs only on the focus point and declaratives.

\section{c) Consciousness}

In the above, we have explained the accent realization and effect of prosody of Iranian learners of Japanese. In this section, we will consider the learners' accent realization from the viewpoint of consciousness. The focus of environment 1 is the part that conveys the important information of the sentence, but it is also the starting point of the conversation sentence in the order of presentation. In this environment, the learners' degree of consciousness is higher than for the whole conversation. For this reason, students are conscious of words and pronunciation, so L1 interference is less likely to occur (Min 1989). On the other hand, in environments 2 and 3, learners have no place to speak emphatically, and gradually tend to allocate attention to the content of utterances, as the degree of consciousness of pronunciation is lower than for environment 1. Therefore, L1 interference becomes strong. The relationship between consciousness and realization of accent is also indicated in Min (1989), and when the learner consciously pronounces it, a change in pitch appears. However, because it violates the accent rule of Japanese, it is not like Japanese.

\section{Conclusion}

In this study, 19 students with a basic proficiency level were targeted, and two hypotheses were developed to verify the accent realization tendency and influence of prosody in units larger than words. As a result of the investigation, Hypothesis 1, that different accent tendencies will emerge for AP receiving sentence accent (when the prosodic environment is strong) and AP not receiving sentence accent (in cases where the prosody is neutral or weak), was verified. Specifically, in the case of the focal environment, the stress accent characteristics are likely to appear, while in other circumstances, the accent tends to be flat. Furthermore, although learners are conscious of accent, their pronunciation may be influenced by the intonation of a phrase or sentence ending.

Hypothesis 2 was that "Persian accent rules will be applied in regard to word accent so that accents are placed at the end of the word." The study found that in the focus environment, other factors than L1 interaction affected learners' accent realization. In the past, we have noted the use of L1 interference in accent realization as well as the interlanguage strategy used by learners. However, in this study, it became clear that the influence of each factor varies depending on the environment. Specifically, in the case of a focus environment, since learners' consciousness of accent is high, a strategy other than L1 interference is used. On the other hand, if there is no focus, the learners' accent consciousness level is low, and the influence of $L 1$ is clearly evident.

The target environments in this research differed in terms of sentence position (i.e., environment 2 in the middle of a phrase, environment 2 leftmost of phrase) and sentence type (i.e., declarative or interrogative). In future work, we will further examine learners' realization of accents / intonation of learners' words by unifying the prosodic environment. In addition, as a result of using only three-mora non-words, LHL occurred more frequently. In the next step, we will investigate how the realization of accent and intonation changes depending on the number of morae. 
$\widehat{1}$
0
0
0
0
0
0
0
0
0
0
0
0
0
0

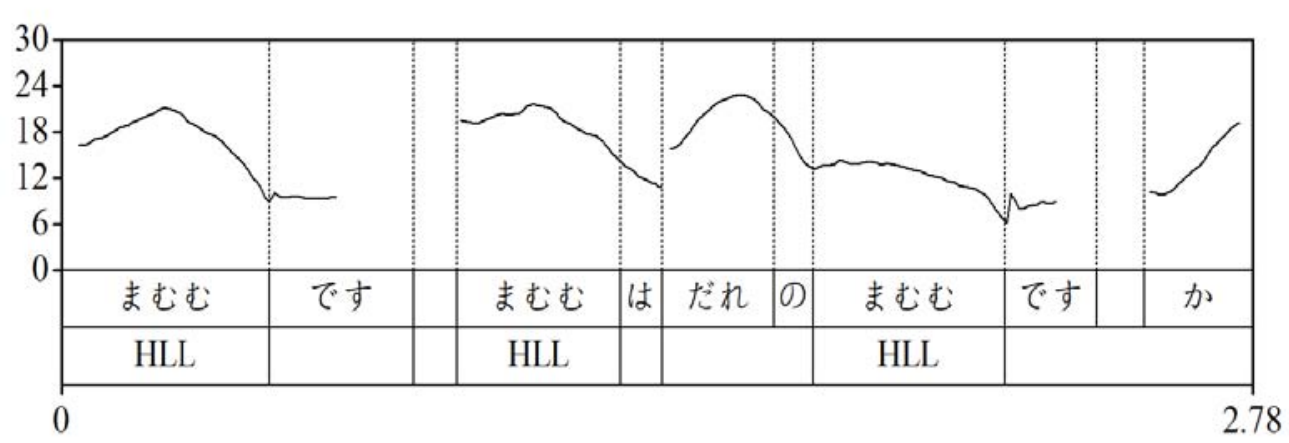

Time (s)

Figure 1: Utterance by native speaker (Female) $(\mathrm{H})$

\begin{tabular}{l}
\multirow{1}{*}{} \\
0 \\
0 \\
$\vdots$ \\
0 \\
0 \\
0 \\
0 \\
0 \\
0 \\
0 \\
0 \\
0
\end{tabular}

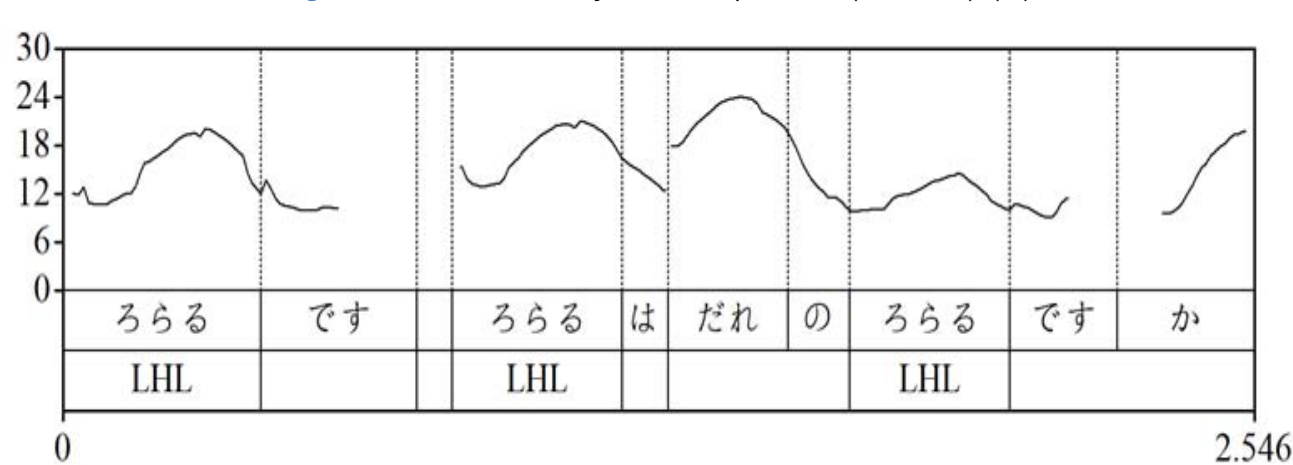

Time (s)

Figure 2: Utterance by native speaker (Female) (LHL)

ปี
0
0
0
0
0
0
0
0
0
0
0
0
0

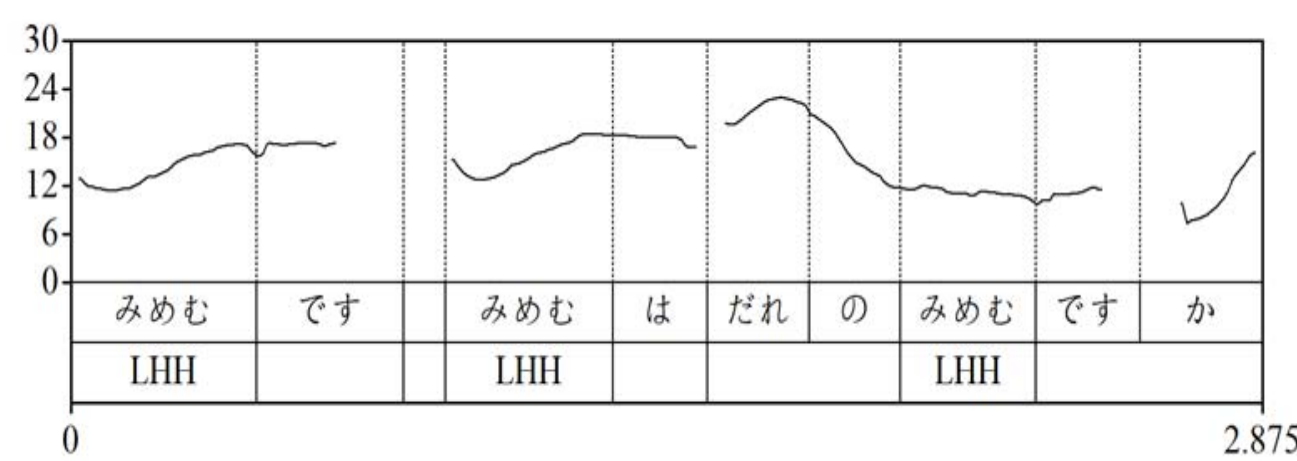

Time (s)

Figure 3: Utterance by native speaker (Female) (LHH)

Nิ
0
8
0
0
0
0
0
0
.0
0
0
0
0
0
0

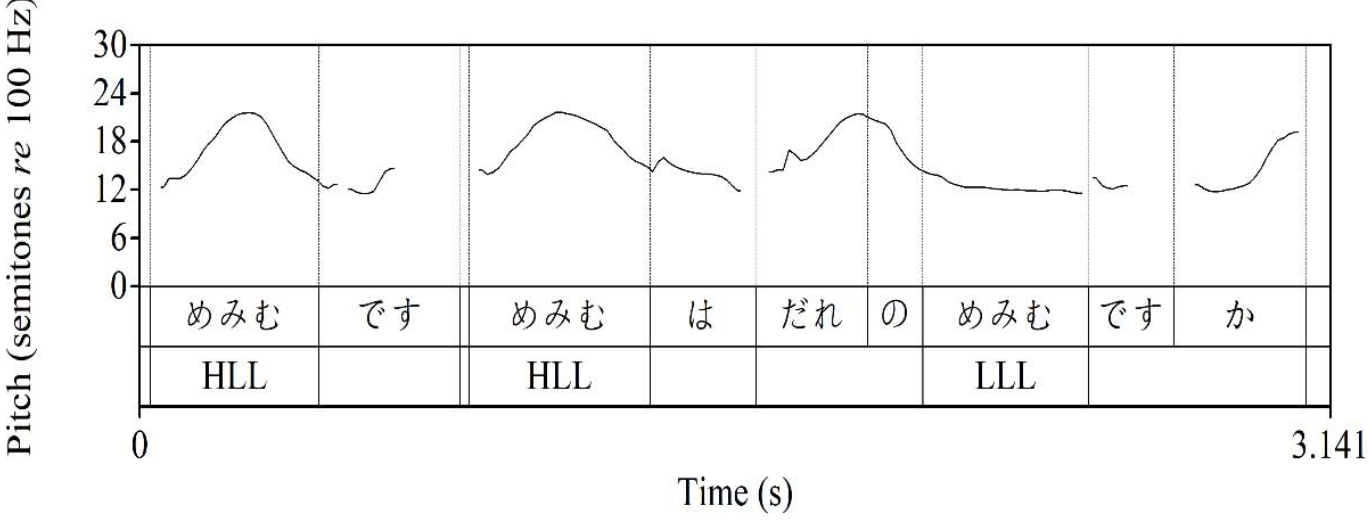

Figure 4: Utterance by S1 (Female) 


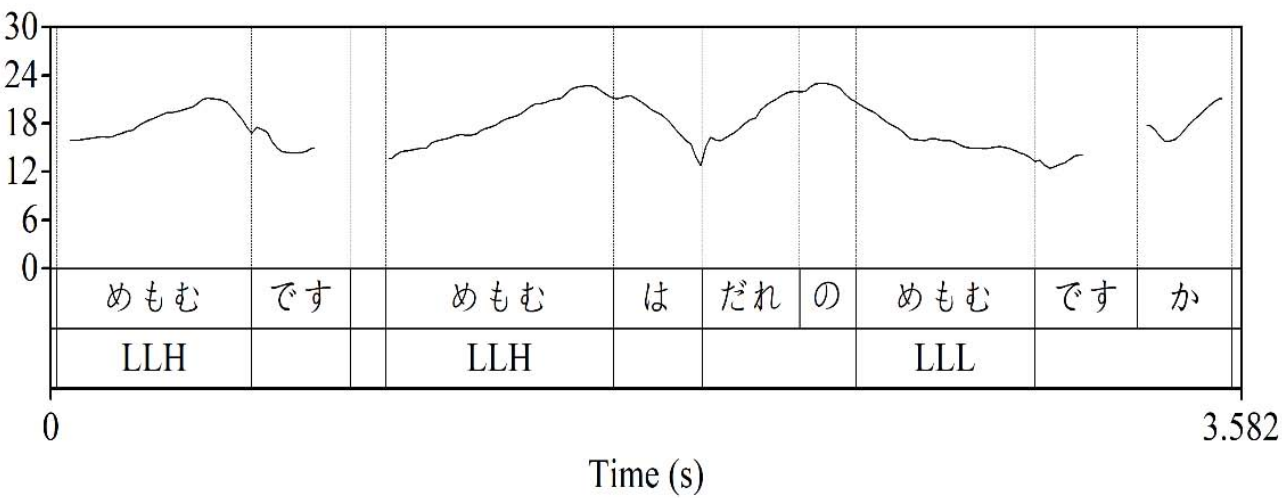

Figure 5: Utterance by S2 (Female)

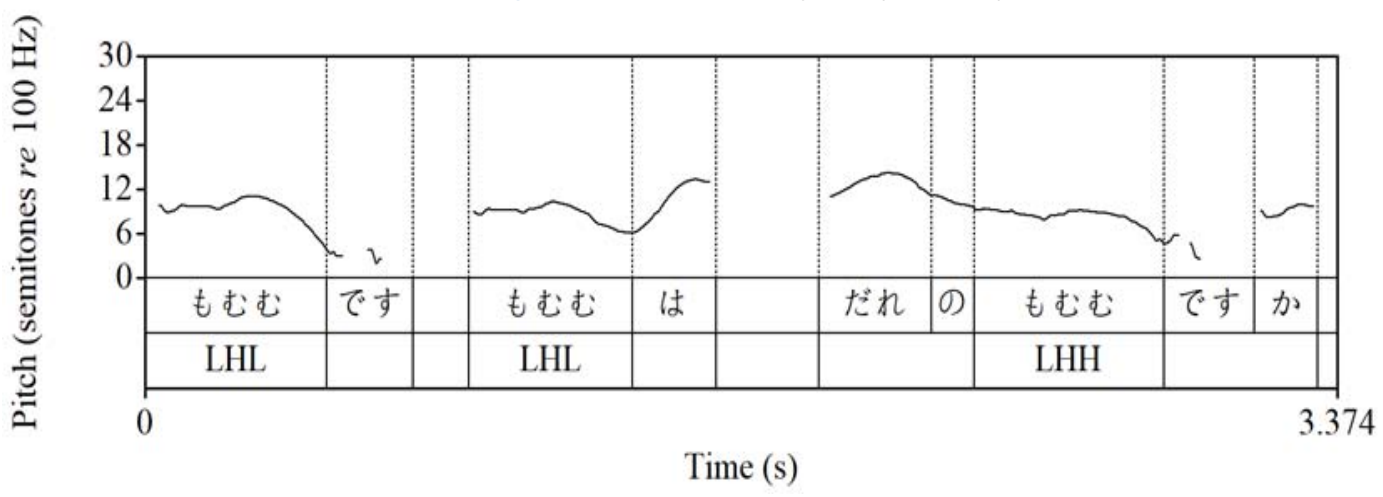

Figure 6: Utterance by S3 (Male)

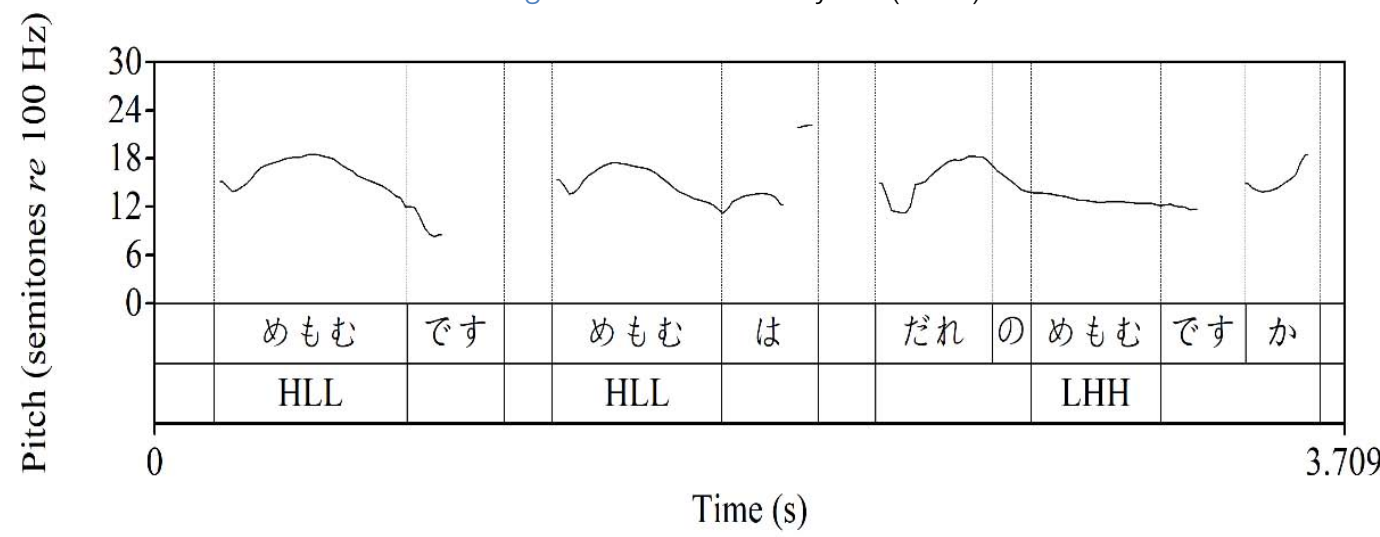

Figure 7: Utterance by S4 (Female)

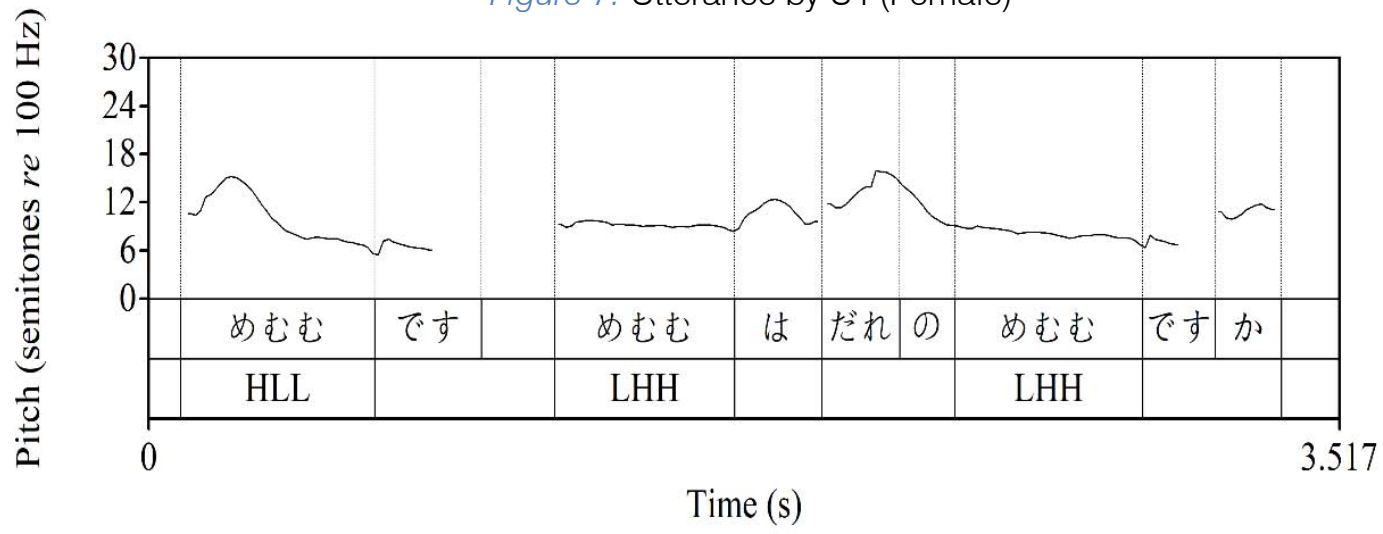

Figure 8: Utterance by S5 (Male) 
N1
$\mathbf{1}$
0
0
0
0
0
0
0
0
0
0
$\frac{1}{0}$
.
0

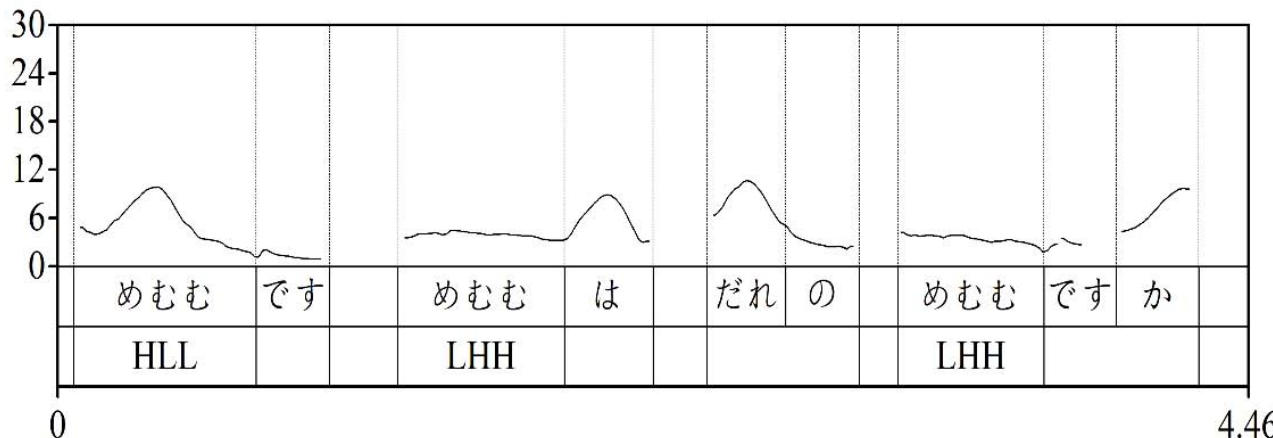

Time (s)

Figure 9: Utterance by S6 (Male)

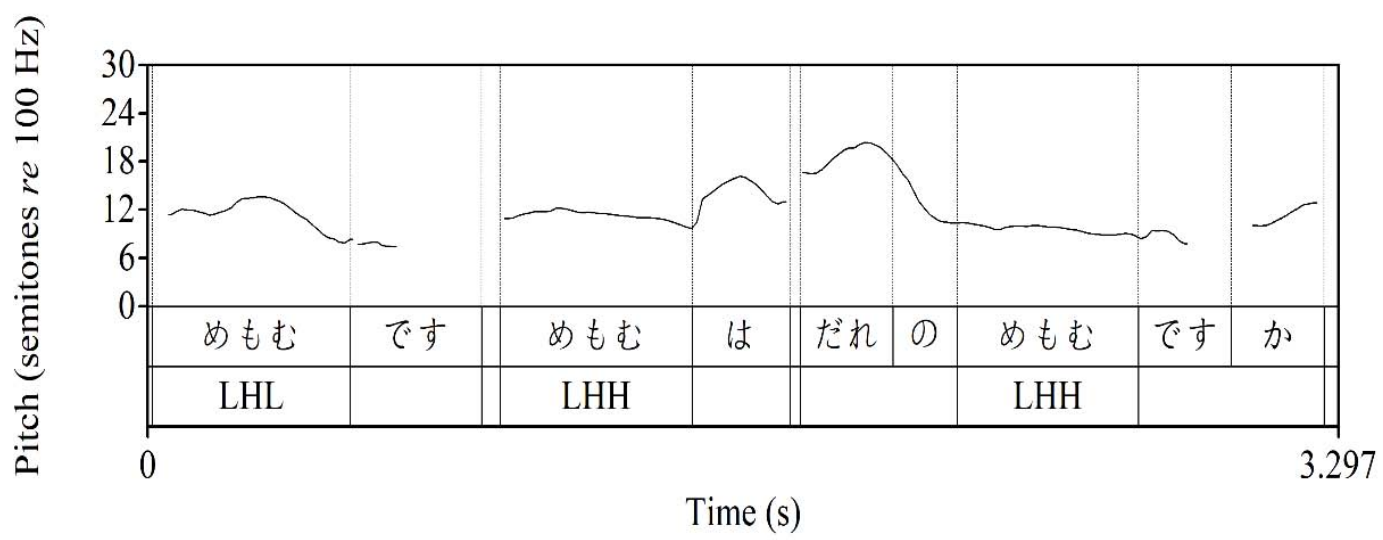

Figure 10: Utterance by S7 (Female)
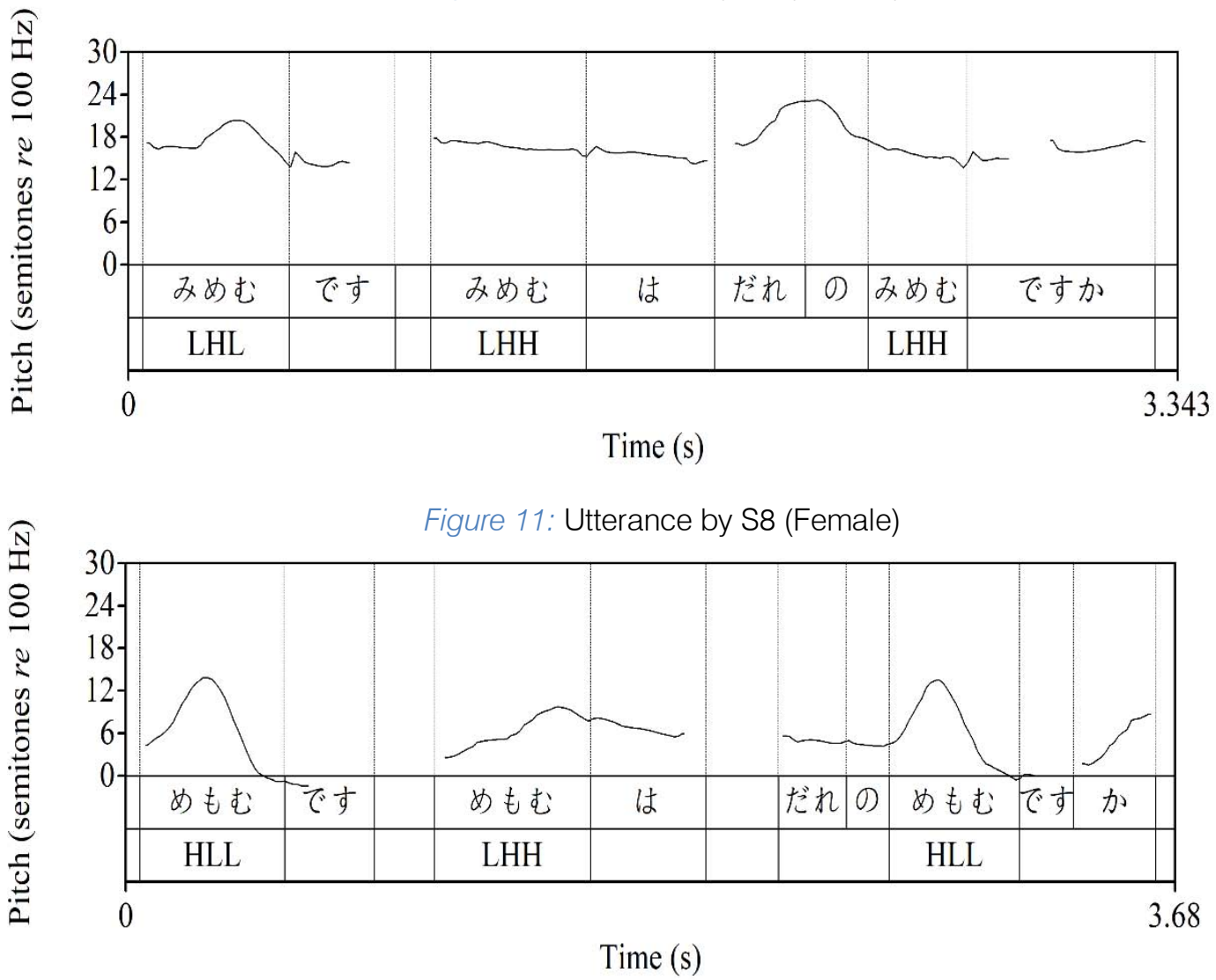

Figure 12: Utterance by S9 (Mmale) 


\section{References Références Referencias}

1. Ayusawa, Takako. (1991) Intonation and Japanese language teaching. Japanese Language Linguistics, 10 (7): 98-113.

2. Ayusawa, Takako. (1993) Intonation of learners of Japanese - acquisition of intonation of the Tokyo Japanese interrogatives -. In: Open Symposium of University and Science Organizing Committee. Internationalizing Japanese: Spoken Language Science and Speech Education, Tokyo: Kubapuro, 165-172.

3. Ayusawa, Takako. (2003) Acquisition of Japanese accents and intonation by foreign learners. Journal of the Phonetic Society of Japan, 7(2): 47-58.

4. Boersma, P., \& Weenink, D. (2015) Praat: Doing phonetics by computer (Version 5.1.25) [Computer program]. Retrieved from http://www.praat.org/.

5. Kori, Shiro. (1997) Japanese intonation - type and function-, In: Sugitou, Miyoko, Kunihiro, Tetsuya, Kowano, Morio, Hirose, Hajime. Accent, Intonation, Rhythm and Pause, Tokyo: Sanshodo, 69-199.

6. Kori, Shiro. (2004) A new analysis of accent in Tokyo Japanese: About handling rise of the beginning of the word. Japanese Language Studies, 55(2): 16-31.

7. Kori, Shiro. (2008) Accent reduction and restrictive modification in Tokyo Japanese. Journal of the Phonetic Society of Japan, 12(1): 34-53.

8. Kori, Shiro. (2012) Restrictive modification and realization of lexical accents in Tokyo Japanese: A quantitative study. Journal of Language and Culture Studies, 38: 1-22.

9. Kubono, Haruo, and Ota, Satoshi. (1998) Phonological Structure and Accent. Tokyo: Kenkyusha.

10. Matsuzaki, Hiroshi and Kawano, Toshiyuki (2010) Phonetics for Succeeding with Japanese Language Education Proficiency Test, Tokyo: Aruku.

11. Matsuzaki, Hiroshi. (2001) Speech education in Japanese. In: Jouo, Hakutaro. Japanese Education Series Volume 3 Computer Phonetics, Tokyo: Oufuu, 207-258.

12. Min, Mitsujun. (1989) Prosodic feature in Japanese speech of Korean speaker and its evaluation by Japanese speaker. Journal of Japanese Language Teaching, 68: 175-190.

13. Nakato, Yasue. (2001) Pitch realization of Korean learners of Japanese in word reading. Journal of Japanese Language Teaching, 109: 80-89.

14. Pierrehumbert, J. B., and Beckman, M. E. (1988) Japanese Tone Structure. Cambridge, MA \& London: MIT Press.

15. Sadat-Tehrani, Nima. (2007) The Intonational Grammar of Persian. Ph.D. dissertation, University of Manitoba.

16. Sukegawa, Yasuhiko. (1999) Pitch realization of 2/3mora-words by Brazilian learners of Japanese.
Journal of the Phonetic Society of Japan, 3(3): 1325.

17. Yamada, Nobuko. (1994) Steps in the acquisition of Japanese lexical accent: The case of foreign learners. Journal of Japanese Language Teaching, 85: 108-120. 